\title{
ORIGINAL ARTICLE \\ Intermittent self-catheterization in tetraplegic patients: a 6-year experience gained in the spinal cord unit in Prague
}

\author{
J Kriz and Z Relichova
}

Objectives: To present a system of urological care for patients with cervical spinal cord injury (SCI) in the Spinal Cord Unit in Prague. Methods: Forty-one out of 412 patients hospitalized with acute SCI between 2007 and 2012 with motor complete SCI (AIS A,B) at the C4-C7 motor level and with sufficient follow-up duration were selected. Patients were trained using a male bladder catheterization model (37 with a transurethral catheter and 4 with a suprapubic catheter) to perform intermittent catheterization (IC) using an ergohand device, and were later encouraged to perform self-catheterization.

Results: On the basis of the motor level of the $\mathrm{SCl}, 8$ out of the 41 study patients were assigned to group 1 (C4), 11 to group 2 (C5), 15 to group 3 (C6) and 7 to group 4 (C7). All patients in group 1 had an indwelling urinary catheter. In group 2, 6 patients (54.6\%) learned to perform IC, with 2 of them needing another person's assistance. In 5 patients (45.5\%), suprapubic cystostomy was maintained (insufficient functional grip, severe autonomic dysreflexia, prompt reflex erection). Group 3 included 12 patients (80\%) performing intermittent catheterization and 3 patients $(20 \%)$ with suprapubic cystostomy (insufficient functional grip, post-bladdersurgery condition, cognitive impairment). In group 4, only 1 patient (14.3\%) had an indwelling catheter due to severe abductor spasticity, whereas the remaining $6(85.7 \%)$ learned to perform IC.

Conclusions: These findings suggest that patients with cervical $\mathrm{SCl}$ below the $\mathrm{C} 5$ motor level are able to learn self-catheterization, which increases independence and decreases the risk of urinary infection and stone formation.

Spinal Cord (2014) 52, 163-166; doi:10.1038/sc.2013.154; published online 17 December 2013

Keywords: spinal cord injury; tetraplegia; autonomic bladder; intermittent catheterization

\section{INTRODUCTION}

Intermittent self-catheterization is undeniably the method of choice for bladder emptying in patients after spinal cord injury (SCI). This method was popularized by Lapides in $1972 .{ }^{1}$ When following specific rules, the method is safe and does not cause serious complications. Patients with an upper motor neuron lesion involving the lower urinary tract most often show detrusor and sphincter hyperactivity with detrusor-sphincter dyssynergia and high-pressure bladder, posing a risk to the upper urinary tract. ${ }^{2}$ Therefore, the need is evident for complete and low-pressure bladder emptying at regular intervals proportional to the natural bladder filling rate. Moreover, patients with SCI above T6 are at high risk of developing autonomic dysreflexia with all negative consequences. ${ }^{3}$

In patients with tetraplegia, upper limb function may be impaired to a variable extent, causing limitations in performing routine daily activities. For the above-mentioned reasons, intermittent urinary catheterization is crucial for this group of patients. While helping individuals with a high spinal cord lesion to achieve maximal independence, it is often counterproductive if urinary catheterization is performed by another person five to six times a day. In the past, triggered micturition was routinely used in such patients and it appeared well tolerated when following certain strict regimens (maximal detrusor pressure during voiding cystometry up to $70-80 \mathrm{~cm} \mathrm{H}_{2} \mathrm{O}$, residuum up to $25 \%$ of volume). However, given the current options for self-catheterization with cervical lesions, the risks of this method do not seem to outweigh the benefits. ${ }^{4}$ Therefore, since 2004, when the Spinal Cord Unit was opened in Prague, all patients have been encouraged to switch to intermittent catheterization (IC) whenever possible. Nevertheless, this article presents the system of urinary tract care for patients with tetraplegia starting in the acute phase of SCI.

\section{PATIENTS AND METHODS}

Between 2007 and 2012, 412 patients with acute SCI were admitted to the Spinal Cord Unit. Of them 143 patients presented with cervical SCI. On the basis of the International Standards for the Neurological Classification of Spinal Cord Injury criteria, ${ }^{5}$ all patients with C4-C7 motor complete SCI (AIS A,B) were enrolled in this study to make it possible to compare the outcomes in different groups in relation to the preserved function of the key upper extremity muscles. At least a 6-month (in 2012 cases) or a 12-month post-injury follow-up at the outpatient clinic was required. The average time of follow-up was 2.8 years. The study cohort included 41 patients (39 men and 2 women). None of the patients lived completely independently. They either had a family caregiver or used services providing personal assistance.

The average interval between the admission to the Spine Surgery Department and a referral to the Spinal Cord Unit was 11 days. The average stay in the Spinal Cord Unit was 78 days and consisted of intensive therapeutic, nursing and rehabilitation care. The upper extremities of patients with residual wrist motion and loss of finger motion were positioned in wrist extension and MP and PIP flexion using special gloves (Figure 1). ${ }^{6}$ Thus, deep finger flexors were shortened, with the fingers placed in position for a cylindrical grip during wrist extension. 
Given that with a permanent urinary catheter inserted on a long-term basis the S-shaped urethra in men involves an increased risk for pressure sores and urethral strictures, the standard indwelling transurethral catheter can be left in place for a maximum of 1-2 weeks. If switching to IC in such a short interval of time was not possible for any reason, suprapubic cystostomy was performed. IC by another person (caregiver or a nurse) was not used because of the fact that patients would revert to a transurethral permanent catheter in order to avoid extreme dependence on a particular caregiver. All patients were transferred to a rehabilitation institute and later discharged home.

Patients with tetraplegia underwent training to perform self-catheterization during their stay in the Spinal Cord Unit. The training was administered daily with a male bladder catheterization model using an ergohand device (Figure 2). First, dry, lubricated or pre-coated hydrophilic catheters were used. The training lasted for approximately 1-2 weeks. When catheterization skills were acquired on the model, patients self-catheterized using a closed catheter (a catheter with a plug) while suprapubic cystostomy was maintained. At the same time, anticholinergic therapy was usually started. If culture positive, the patient was switched to IC with antibiotic protection. Finally, when the patients were able to self-catheterize independently, suprapubic cystostomy was removed. All patients were instructed to use other catheterization aids, especially a penis holder, a cloth holder and aids to spread the legs apart but most importantly they were instructed on the use of adapted clothing, especially underwear.

Some patients needed additional training or had to wait until their upper extremity function had improved. Cognitive screening was performed whenever cognitive dysfunction was suspected by the clinician.

Subsequently, all patients in the study were referred to a rehabilitation center to continue rehabilitation for an additional 4-5 months before being discharged home. On the basis of the level of SCI, the patients were divided into four groups (C4, C5, C6 and C7). All patients were contacted regularly for in-person follow-up exams and were assessed at the Spinal Cord Unit and at Urology sections of respective hospitals.

All patients underwent a urodynamic examination between 4 and 6 months after the injury, and medication was adjusted on the basis of the results. A

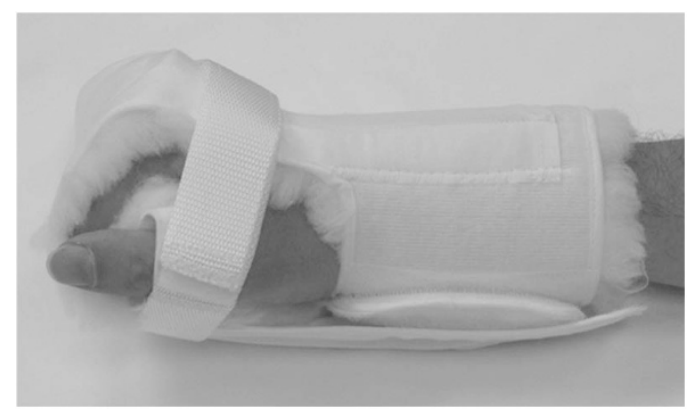

Figure 1 Positioning glove for a tetraplegic hand.

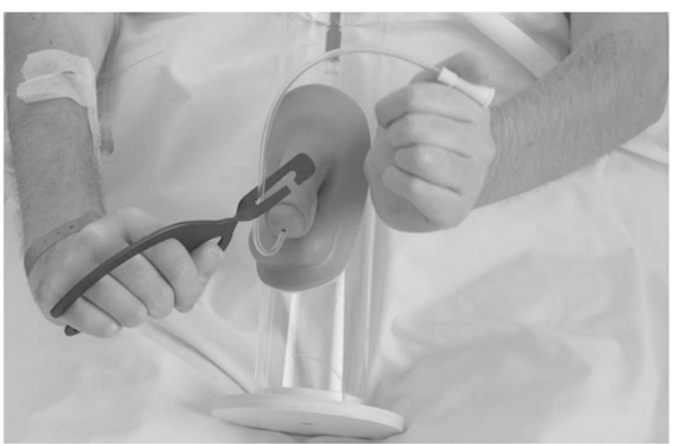

Figure 2 Training on a male bladder catheterization model using an ergohand device. follow-up exam was performed 1 year and 2 years after the injury at the SCI clinic. Further standard follow-ups were administered in a urological clinic in the area of the patient's residence with yearly consultation at a urology clinic and, if needed, in an outpatient SCI center.

In addition to detailed anamnesis, urological examination includes urodynamics outcomes, ultrasonography and other imaging methods. Full medical history was obtained, including specific urological questions. The focus was on the urinary diversion method used and, if IC was not possible, on the reason why. The patients were asked about their pre-catheterization history, compliance with the training program utilizing the prescribed devices, ability to self-catheterize while in any position, use of any devices, problems with urinary leakage, the use of any incontinence products, type of urinary catheters and urine bags, occurrence of spasticity and autonomic dysreflexia, catheterization intervals, perception of bladder filling and use of urological medication. The overall quality of life is not a part of the standard assessment at present.

\section{RESULTS}

Eight $(19.5 \%)$ out of the 41 patients sustained a C4 level of SCI (group 1), $11(26.8 \%)$ a C5 level of SCI (group 2), $15(36.6 \%)$ a C6 level of SCI (group 3) and 7 (17.1\%) a C7 level of SCI (group 4) (Table 1). The sample included only two women: one in group 1 and one in group 2. The leading cause of SCI was diving in shallow water $(51.2 \%)$, followed by car accidents $(26.8 \%)$. Other causes were falls and motorcycle and bicycle accidents.

\section{Group 1 (C4)}

Seven patients had an indwelling suprapubic catheter and one female patient had an indwelling transurethral catheter. The mean age of the patients was 44 years. Wrist extensor muscle strength varied between grades 0 and 1 in all subjects, but one female patient presented with grade 3 unilateral wrist extensor muscle strength.

\section{Group 2 (C5)}

The mean age of the patients in group 2 was 34 years. Six (54.6\%) out of 11 patients in group 2 were using IC, with two of them needing another person's assistance to perform the procedure. Five patients had an indwelling suprapubic catheter. The reasons for continuing to use indwelling urinary catheters were as follows: failure to develop a functional grip in one patient, repetitive severe autonomic dysreflexia occurring during the catheterization procedure in one patient, prompt reflex erection in one patient and reduced wrist extensor muscle strength in the other two patients. One of them was a woman at 7 months post injury. The patients performing self-catheterization received training using special devices and had indwelling suprapubic catheters for an average of 14 months.

\section{Group 3 (C6)}

The mean age of the patients in group 3 was 27 years. Twelve $(80 \%)$ out of 15 patients in group 3 achieved the ability of

Table 1 Numbers, mean age and bladder management of subjects according to the motor level of $\mathrm{SCl}$

\begin{tabular}{|c|c|c|c|c|c|c|c|c|c|}
\hline \multirow{2}{*}{ Group } & \multirow{2}{*}{$\begin{array}{c}\text { Motor } \\
\text { level }\end{array}$} & \multirow{2}{*}{$\mathrm{N}$} & \multirow{2}{*}{$\begin{array}{c}\text { Age } \\
\text { Mean } \pm \text { s.d. }\end{array}$} & \multicolumn{2}{|c|}{ Indw. catether } & \multicolumn{2}{|c|}{ Asisted IC } & \multicolumn{2}{|c|}{ Independent IC } \\
\hline & & & & $\mathrm{N}$ & $\%$ & $\mathrm{~N}$ & $\%$ & $\mathrm{~N}$ & $\%$ \\
\hline 1 & C4 & 8 & $45.1 \pm 17.0$ & 8 & 100 & 0 & 0 & 0 & 0 \\
\hline 2 & C5 & 11 & $34.6 \pm 8.1$ & 5 & 45.5 & 2 & 18.2 & 4 & 36.4 \\
\hline 3 & C6 & 15 & $27.8 \pm 5.7$ & 3 & 20 & 2 & 13.3 & 10 & 66.7 \\
\hline 4 & $\mathrm{C7}$ & 7 & $29.4 \pm 9.1$ & 1 & 14.3 & 0 & 0 & 6 & 85.7 \\
\hline
\end{tabular}

Abbreviations: IC, intermittent catheterization; SCI, spinal cord injury. 
self-catheterization, and two patients needed another person's assistance to perform the procedure in the supine position. Three patients remained with indwelling suprapubic catheters for the following reasons: failure to achieve a functional grip, repeated bladder surgery and cognitive impairment. Patients who were able to perform selfcatheterization had suprapubic cystostomy for an average of about 7 months and all of them were trained in a standard way.

\section{Group 4 (C7)}

The mean age of the patients in group 4 was 29 years. All but 1 patient $(85.7 \%)$ with a C7 level of SCI acquired self-catheterization skills. One patient required an indwelling suprapubic catheter because of severe abductor spasticity and consequent inability to perform selfcatheterization. Four out of the seven patients in group 4 had a cystostomy for an average of 4 months, and two patients had an indwelling transurethral catheter for a short period of time. Three patients did not need training using special devices.

Only $20.8 \%$ of the patients performing self-catheterization continued using the ergohand device. None of the patients used the other catheterization aids. Nevertheless, all patients wore adapted clothes (extended zipper). Altogether, $79.2 \%$ and $20.8 \%$ of patients used hydrophilic and lubricated catheters, respectively. In all, $83.3 \%$ of patients performing IC were able to perceive bladder filling alternatively, with the average volume of catheter-collected urine being $385 \mathrm{ml}$. A total of $70.8 \%$ of patients were using anticholinergic drugs; one patient was given botulinum toxin. Sporadic urinary leakage because of bladder overfilling ( $5 \mathrm{x}$ per week max) and the use of incontinence products were reported by $41.7 \%$ of patients. All of them were using anticholinergic drugs based on the results of a urodynamic assessment. No significant difference in the incidence of autonomic dysreflexia was found between groups 2 and 4 for patients performing intermittent self-catheterization. All patients who started to self-catheterize after the injury continued to use this method even at the end of the study.

\section{DISCUSSION}

Intermittent self-catheterization is currently the gold standard of care for patients with autonomous urinary bladder. ${ }^{7}$ In patients with paraplegia, there is practically no need for alternative methods of bladder emptying, apart from poor compliance or an anatomical obstacle. While in the Spinal Cord Unit, the patients are encouraged to learn to perform self-catheterization to promote their independence as much as possible. Nevertheless, patients with tetraplegia are limited mainly by upper extremity motor impairment. Despite this fact, most of them are able to acquire self-catheterization skills while using special devices in a well-adjusted environment. Apart from compliance and motivation, biceps and wrist extensor muscle strength is a crucial factor. ${ }^{8}$ To illustrate the outcomes of the Spinal Cord Unit, patients with tetraplegia with a complete motor SCI were selected and stratified into four groups according to the motor level of injury. In group 2 (C5), particular focus was placed on the wrist muscle strength.

\section{Group 1 (C4)}

Patients with an SCI at the C4 motor level tend to have considerably limited active motion of the elbow and often zero active motion of the wrist and are not expected to be able to perform self-catheterization. Seven patients had an indwelling suprapubic catheter and one female patient had an indwelling transurethral catheter. We are aware of the risks involved with a long-term indwelling urinary catheter, such as increased risk for a lower urinary tract infection or a bladder stone formation. ${ }^{4}$

Other urinary diversion options include surgical procedures such as urinary sphincterotomy with subsequent use of a urinal, or an incontinent Bricker urinary diversion by ureteroileostomy, initially designed for patients with cancer. Nevertheless, these surgical procedures have not been widely used for this indication in the Czech Republic.

\section{Group 2 (C5)}

In our opinion, and based on the results of the present study, the C5 motor level represents the highest level of SCI for self-catheterization. Grade 3 or greater wrist extensor muscle strength is vital. The most common reason for not including these patients in the group with a C6 level SCI is that, at least unilaterally, brachial biceps muscle strength is grade 4 or wrist extensor muscle strength is grade 2 on the worse side. ${ }^{8}$ Six patients in this group preferred IC, whereas five patients continued to use an indwelling suprapubic catheter. Nevertheless, 8 months after injury, two patients performing IC still had to undergo cystostomy as a rescue option. Two patients needed another person's assistance to perform catheterization. This group includes the second female patient from the overall number of female patients in our sample. Because of the lack of strength of wrist extensors, she was not able to perform self-catheterization at the time of assessment (only 7 months post injury). Nonetheless, we expect improvement in function. At present, a female catheterization model is available, which could be used for this specific patient.

\section{Group 3 (C6)}

In this group, all patients should be able to self-catheterize either with or without special devices, with some of them needing another person's assistance while dressing, using incontinence products or in some positions. Nevertheless, 3 out of 15 patients in this group had an indwelling suprapubic catheter. One of them had a poor functional grip, one underwent repeated bladder surgery and one patient was unable to self-catheterize because of cognitive impairment. Patients performing intermittent self-catheterization used an indwelling suprapubic catheter for a slightly shorter period of time.

In both C5 and C6 motor levels, there is a possibility of improving hand function and self-catheterization ability through upper limb reanimation. Upper limb reanimation protocols consist of reconstructive surgery combined with rehabilitation procedures. ${ }^{9}$

\section{Group 4 (C7)}

The last group included seven patients with an SCI at the C7 level. Only one of them was unable to self-catheterize because of high adductor spasticity and still has a cystostomy, although another option has been considered for him.

All patients performing intermittent self-catheterization avoided excessive bladder filling and self-catheterized at reasonable intervals. On ultrasonography, none of the patients were seen to have any severe urological complications.

Invasive surgical treatment methods should be considered for patients who are unable to self-catheterize even with the use of aids due to either upper extremity motor deficit, lower extremity spasticity or a cognitive deficit. This should be considered on an individual basis. Sphincterotomy with triggered micturition with the use of urinary or incontinence aids is the most conservative procedure. Such patients require consistent urological follow-up with urodynamic checks. Complications linked to autonomic dysreflexia pose a special indication for such a procedure. Urinary bladder augmentation with 
modification according to Mitrofanoff or Monti can be considered for patients with at least partially preserved motor function, especially for women. Ureteroileostomy according to Bricker can be considered in patients with severe cognitive dysfunction or impairment. Alternative methods for certain patients include sacral neurostimulation or dorsal rhizotomy with stimulation of dorsal spinal roots (Brindley procedure). The use of these methods in the Czech Republic is in its beginning stages. However, it is only a matter time before its implementation increases.

\section{CONCLUSION}

Achieving self-catheterization is vital to increasing independence in patients after an SCI. The procedure is being associated with a low risk for urinary tract impairment. Patients who do not need another person's assistance are more independent while at home, work or when traveling.

In this study, we presented bladder management in patients with cervical SCI and found that these patients were able to learn to selfcatheterize when their spinal lesion was below the C5 motor level. Positioning the upper limbs to achieve a functional grip and subsequent simulation training in self-catheterization using an ergohand device during the stay in the Spinal Cord Unit are important steps toward self-catheterization.

\section{DATA ARCHIVING}

There were no data to deposit.

\section{CONFLICT OF INTEREST}

The authors declare no conflict of interest.

\section{ACKNOWLEDGEMENTS}

The study was supported by 'Movement without Help' foundation.

1 Lapides J, Diokno AC, Silber SJ, Lowe BS. Clean, intermittent self-catheterization in the treatment of urinary tract disease. J Urol 1972; 107: 458-461.

2 Samson G, Cardenas DD. Neurogenic bladder in spinal cord injury. Phys Med Rehabil Clin N Am 2007; 18: 255-274.

3 Krassioukov A, Warburton DE, Teasell R, Eng JJ. Spinal Cord Injury Rehabilitation Evidence Research Team. A systematic review of the management of autonomic dysreflexia after spinal cord injury. Arch Phys Med Rehabil 2009; 90 682-695.

4 Wyndaele JJ, Madersbacher H, Kovindha A. Conservative treatment of the neuropathic bladder in spinal cord injured patients. Spinal Cord 2001; 39: 294-300.

5 Kirshblum SC, Waring W, Biering-Sorensen F, Burns SP, Johansen M, Schmidt-Read M et al. Reference for the 2011 revision of the International Standards for Neurological Classification of Spinal Cord Injury. J Spinal Cord Med 2011; 34: 547-554.

6 Doll U, Maurer-Burkhard B, Spahn B, Fromm B. Functional hand development in tetraplegia. Spinal Cord 1998; 36: 818-821.

7 Di Benedetto P. Clean intermittent self-catheterization in neuro-urology. Eur J Phys Rehabil Med 2011; 47: 651-659.

8 Asayama K, Kihara K, Shidoh T, Shigaki M, Ikeda T. The functional limitations of tetraplegic hands for intermittent clean self-catheterisation. Paraplegia 1995; 33 30-33.

9 Bernuz B, Guinet A, Rech C, Hugeron C, Even-Schneider A, Denys P et al. Selfcatheterization acquisition after hand reanimation protocols in $\mathrm{c5}-\mathrm{c} 7$ tetraplegic patients. Spinal Cord 2011; 49: 313-317. 